\title{
Next-to-Leading Order Jet Physics with BLACKHAT
}

\author{
C. F. Berger ${ }^{a}$, Z. Bern ${ }^{b}$, L. J. Dixon ${ }^{c}$, F. Febres Cordero ${ }^{d}$, D. Forde ${ }^{e, f}$, T. Gleisberg ${ }^{c}$, \\ H. Ita ${ }^{b}$, D. A. Kosower ${ }^{g *}$ and D. Maître ${ }^{h *}$ \\ ${ }^{a}$ Center for Theoretical Physics, MIT, Cambridge, MA 02139, USA \\ ${ }^{b}$ Department of Physics and Astronomy, UCLA, Los Angeles, CA 90095-1547, USA \\ ${ }^{c}$ SLAC National Accelerator Laboratory, Stanford University, Stanford, CA 94309, USA \\ ${ }^{d}$ Universidad Simón Bolívar, Departamento de Física, Apartado 89000, Caracas 1080A, \\ Venezuela \\ ${ }^{e}$ Theory Division, Physics Department, CERN, CH-1211 Geneva 23, Switzerland \\ ${ }^{f}$ NIKHEF Theory Group, Science Park 105, NL-1098 XG Amsterdam, The Netherlands \\ ${ }^{g}$ Institut de Physique Théorique, CEA-Saclay, F-91191 Gif-sur-Yvette cedex, France \\ ${ }^{h}$ Department of Physics, University of Durham, DH1 3LE, UK
}

\begin{abstract}
We present several results obtained using the BLACKHAT next-to-leading order QCD program library, in conjunction with SHERPA. In particular, we present distributions for vector boson plus 1,2,3-jet production at the Tevatron and at the asymptotic running energy of the Large Hadron Collider, including new $Z+3$-jet distributions. The $Z+2$-jet predictions for the second-jet $P_{T}$ distribution are compared to CDF data. We present the jet-emission probability at NLO in $W+2$ jet events at the LHC, where the tagging jets are taken to be the ones furthest apart in pseudorapidity. We analyze further the large left-handed $W^{ \pm}$polarization, identified in our previous study, for $W$ bosons produced at high $P_{T}$ at the LHC.
\end{abstract}

RADCOR 2009 - 9th International Symposium on Radiative Corrections (Applications of Quantum Field Theory to Phenomenology), October 25 - 30 2009, Ascona, Switzerland

\footnotetext{
*Speaker.
} 


\section{Introduction}

The dawn of the Large Hadron Collider (LHC) era brings renewed incentive to continue improving theoretical predictions of Standard-Model backgrounds to new physics searches. For many searches, including some channels for the Higgs boson and for dark matter particles, the signals will be excesses in jet + lepton or jet + missing $E_{T}$ distributions. Such signals can be mimicked by Standard-Model processes; accordingly, a thorough and quantitatively reliable theoretical prediction is needed. This requires a calculation through next-to-leading order (NLO) in QCD.

Leading-order (LO) computations, while an important first step, suffer from a strong dependence on the unphysical renormalization and factorization scales. At this order, they enter only through the strong coupling $\alpha_{s}$ and parton distribution functions, uncompensated by any behavior of the short-distance partonic matrix elements. Because the QCD coupling is large and runs quickly, the absolute normalization of cross sections has a substantial dependence on scales. For reasonable scale variations, the dependence is of the order of $\pm 40 \%$ for the $V+3$-jet processes we shall study, with $V$ a heavy electroweak vector boson. The dependence also grows substantially with increasing number of jets. At NLO, the virtual corrections introduce a compensating dependence on the scales. The scale dependence shrinks to $\pm 10 \%$, and we obtain a quantitatively reliable answer. Shapes of distributions can also show a dramatic scale dependence with poor scale choices. Some shapes do display noticeable "genuine" NLO corrections, independent of scale issues.

NLO predictions for $V+n$-jet production at hadron colliders require several ingredients:

- tree-level $V+(n+2)$-parton matrix elements, which provide the LO contribution;

- interference of one-loop and tree amplitudes for $V+(n+2)$ partons (virtual contribution);

- tree-level $V+(n+3)$-parton matrix elements (real-emission contribution);

- a subtraction approximation capturing the singular behavior of the real-emission term;

- the integral of the approximation over the singular phase space (real-subtraction term).

These contributions must be convoluted with parton distribution functions, obtained from NLO fits, and integrated over the final phase space, incorporating appropriate experimental cuts.

Schematically, we combine the contributions as follows,

$$
\begin{aligned}
\frac{d \sigma_{V+n}^{\mathrm{NLO}}}{d \mathrm{Obs}}=\int d x_{1,2} f_{1} f_{2} & {\left[\int d \Phi_{n} \delta_{\mathrm{Obs}} \sigma_{2 \rightarrow V+n}^{\text {tree }}+\int d \Phi_{n} \delta_{\mathrm{Obs}}\left(\sigma_{2 \rightarrow V+n}^{1-\text { loop }}+\sigma_{2 \rightarrow V+n}^{\int \mathrm{app}}\right)\right.} \\
& \left.+\int d \Phi_{n+1} \delta_{\mathrm{Obs}}\left(\sigma_{2 \rightarrow V+n+1}^{\mathrm{tree}}-\sigma_{2 \rightarrow V+n+1}^{\mathrm{app}}\right)\right]
\end{aligned}
$$

where $d \Phi_{n}$ denotes the $V+n$-parton phase space; $d x_{1,2} f_{1} f_{2}$ the integral over the appropriate parton distributions, a sum over types being implicit; $\delta_{\mathrm{Obs}}$, the binning function for the desired distribution; $\sigma^{\text {tree }}$, the tree-level squared matrix elements; $\sigma^{1-\text { loop }}$, the virtual corrections; $\sigma^{\text {app }}$, the approximation to the real-emission contribution; and $\sigma^{\int}$ app , the approximation's integral over singular phase space. The set of subtraction terms ensures that each of the terms in this equation is separately finite, and thus may be computed numerically. 


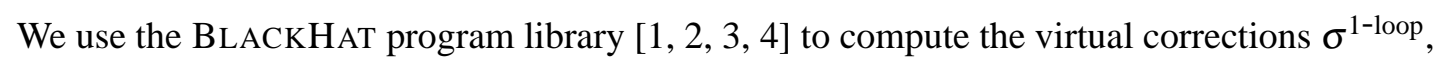
and the SHERPA package [5] to compute $\sigma^{\text {tree }}$ and the required approximation ( $\sigma^{\text {app }}$ and $\sigma^{\int \text { app }}$ ). The approximation uses the Catani-Seymour dipole approach [6]. The phase-space integration is performed with SHERPA, implementing a multi-channel approach [7].

The BLACKHAT library implements on-shell methods for one-loop amplitudes numerically. Such amplitudes can be written as a sum of cut terms $C_{n}$, containing branch cuts in kinematic invariants, and rational terms $R_{n}$, free of branch cuts,

$$
A_{n}=C_{n}+R_{n}
$$

All the branch cuts appear in the form of logarithms and dilogarithms, and can be written as a sum over a basis of scalar integrals — bubbles $I_{2}^{i}$, triangles $I_{3}^{i}$, and boxes $I_{4}^{i}$,

$$
C_{n}=\sum_{i} d_{i} I_{4}^{i}+\sum_{i} c_{i} I_{3}^{i}+\sum_{i} b_{i} I_{2}^{i}
$$

(Massive particles in the loop also require tadpole integrals.) We take all external momenta to be four dimensional, expressible in terms of spinors. The coefficients of these integrals, $b_{i}, c_{i}$, and $d_{i}$, as well as the rational remainder $R_{n}$, are rational functions of spinor variables (in the form of spinor products). The BLACKHAT library computes these coefficients numerically, leveraging off recent analytic progress. In particular, it exploits generalized unitarity $[8,9]$. We use Forde's approach [10] to compute $b_{i}$ and $c_{i}$, making use also of the subtraction approach to integral reduction first introduced by Ossola, Papadopoulos and Pittau [11]. To obtain the rational terms we have implemented both loop-level on-shell recursion [12], and a "massive continuation" approach due to Badger [13], which is related to the $D$-dimensional generalized unitarity [14] approach of Giele, Kunszt and Melnikov [15].

One-loop matrix element computations can suffer from numerical instabilities. In BLACKHAT, this problem is solved by detecting pieces of the amplitude which do not have a sufficient accuracy and recomputing them with higher precision using the multiprecision package QD [16]. This approach has the advantage of solving the problem using the same approach for well-behaved points and for numerically unstable ones. As discussed in refs. [1, 4], with a series of tests - the simplest of which checks whether the infrared divergences have the proper values - there is no need for a priori knowledge of what set of circumstances can lead to instabilities. In each contribution where precision loss is detected, BLACKHAT automatically switches to higher precision, regardless of the underlying cause. With on-shell methods this happens infrequently and therefore has only a mild effect on the overall computation time.

We have previously used these software tools to provide the first phenomenologically useful NLO study of the production of a $W$ boson in association with up to three jets [3, 4]. In this Contribution, we extend our previous studies with a more detailed look at the question of scale choices; at aspects of the polarization of $W \mathrm{~s}$ produced at high $P_{T}$; and at a new distribution displaying the probability of emitting a jet into a rapidity gap. We also present the first NLO results on $Z+3$-jet production at hadron colliders, in a leading-color approximation designed to be accurate within a few percent. In all cases, we decay the vector boson to leptons, $W^{+} \rightarrow l^{+} v_{l}, W^{-} \rightarrow l^{-} \bar{v}_{l}$, and $Z \rightarrow l^{+} l^{-}$, using the appropriate vector boson linewidth. We include the virtual photon contribution to $l^{+} l^{-}$production. Other recent state-of-the-art NLO results may be found in ref. [17]. The 
production of $W+3$ jets has also been computed at NLO using a leading-color approximation and extrapolation $[18,19]$.

\section{Scale Choices}

The renormalization and factorization scales are not physical scales. Physical quantities should be independent of them. A dependence on them is nonetheless present in theoretical predictions that are truncated at a fixed order in perturbation theory. At leading order, the dependence arises solely through $\alpha_{s}$ and the parton distributions, respectively. We adopt the usual practice and choose the two to be equal, $\mu_{R}=\mu_{F}=\mu$. NLO results greatly reduce the dependence compared to LO, but of course they do not eliminate it completely. We still need to choose this scale. We should expect a good choice for $\mu$ to be near a typical energy scale for the observable we are computing, in order to minimize the uncomputed logarithms in higher-order terms. However, multi-jet processes such as $V+2,3$-jet production have many intrinsic scales, and it is not clear a priori how to distill them into a single number. For any given point in the fully-differential cross section, there is a range of scales one could plausibly choose. For example, one might choose the same fixed scale $\mu$ for all events. However, because there can be a large dynamic range in momentum scales (particularly at the LHC, where jet transverse energies well above $M_{W}$ are common), it is natural to pick the scale $\mu$ dynamically, event by event, as a function of the event's kinematics.
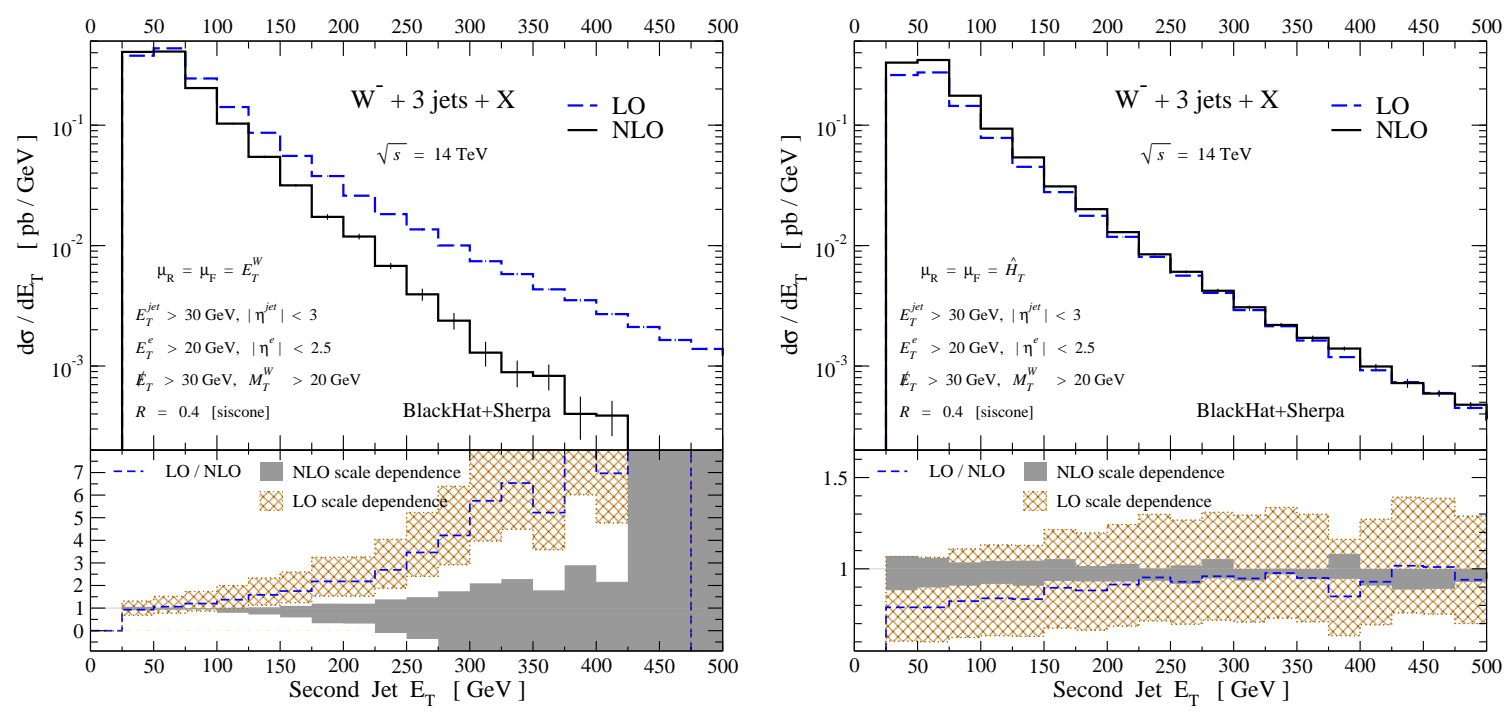

Figure 1: LO and NLO predictions for the second jet $E_{T}$ distribution in $W+3$ jet production at the LHC. The only difference between the left and right panels is the scale choice: $\mu=E_{T}^{W}$ on the left and $\mu=\hat{H}_{T}$ on the right. The former choice is clearly problematic and should not be used in phenomenological studies. The bottom panels show the LO and NLO predictions, varied by a factor of two around the central scale, and divided by the NLO value at the central scale.

Previous studies (see e.g. refs. [20, 21]) have used the transverse energy of the vector boson, $E_{T}^{V}$, as the scale choice. For many distributions at the Tevatron, this is satisfactory. With the larger dynamic range at the LHC, the choice becomes problematic. Indeed, for some observables, such as the transverse-energy distribution of the second-hardest jet in $W+3$-jet production, shown in the 

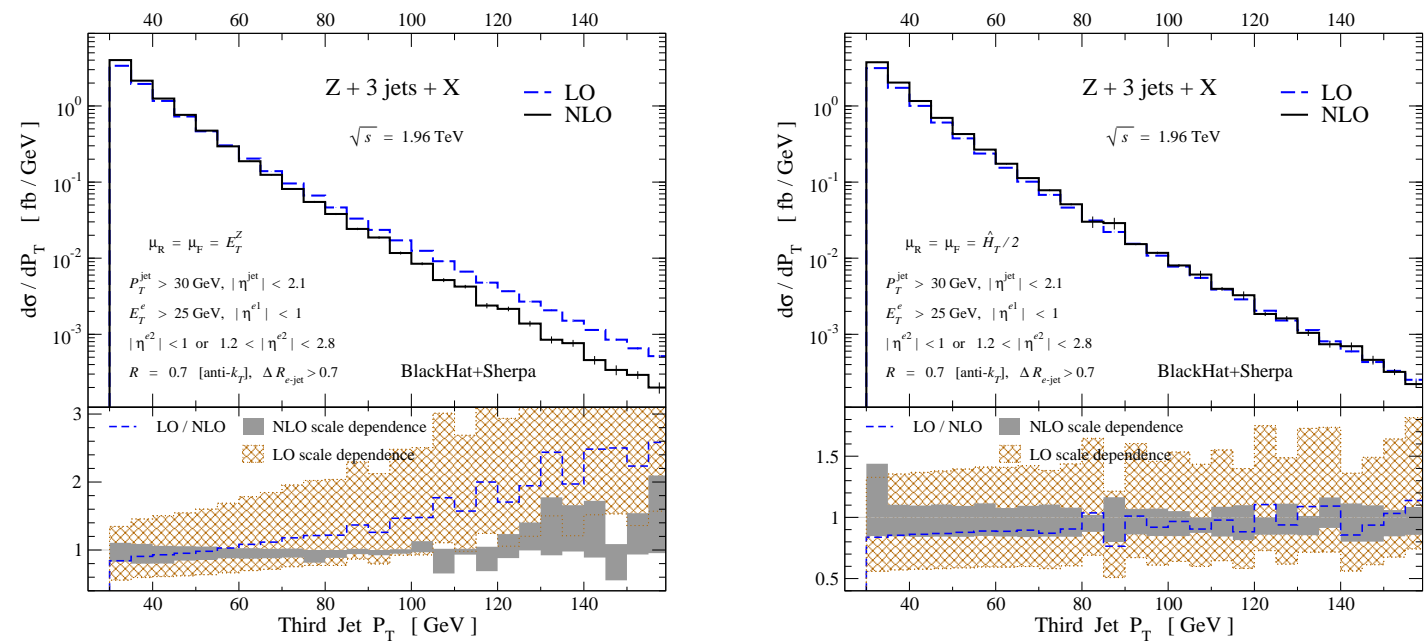

Figure 2: The NLO $P_{T}$ distribution of the third jet in $Z+3$-jet production at the Tevatron. For the left panel the scale choice $\mu=E_{T}^{Z}$ is used, and for the right panel $\mu=\hat{H}_{T} / 2$. Although the two NLO results are compatible, the LO results have large shape differences, illustrating that $\mu=\hat{H}_{T} / 2$ is a better choice than $\mu=E_{T}^{Z}$ at the Tevatron as well. The lepton and jet cuts match the CDF ones [20].

left panel of fig. 1, it goes disastrously wrong, leading to negative values of the distribution for $E_{T}$ beyond $475 \mathrm{GeV}$. Even at the Tevatron, the scale choice $\mu=E_{T}^{V}$ is not necessarily a good one; for example, with this choice, the left panel of fig. 2 displays a large change in shape between LO and NLO in the $P_{T}$ distribution of the third hardest jet in $Z+3$-jet production. This difficulty reflects the emergence of a large logarithm $\ln (\mu / E)$, where $E$ is a typical energy scale, spoiling the validity of the perturbative expansion.

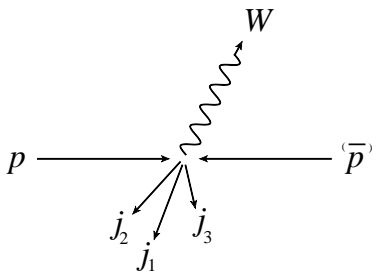

(a)

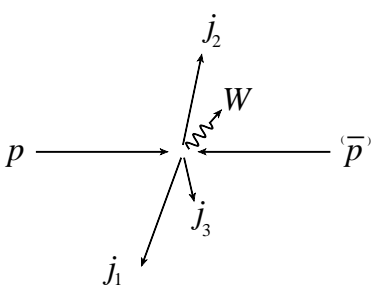

(b)

Figure 3: Two distinct $W+3$ jet configurations with rather different values for the $W$ transverse energy. In configuration (a) an energetic $W$ balances the energy of the jets, while in (b) the $W$ is relatively soft. Configuration (b) generally dominates over (a) when the jet transverse energies get large.

To understand the problem with the scale choice $\mu=E_{T}^{V}$, consider the two configurations depicted in fig. 3. In configuration (a), the $W$ has a transverse energy larger than that of the jets, and accordingly sets the scale for the process. In configuration (b), the two leading jets roughly balance in $E_{T}$, while the $W$ has much lower transverse energy. Here, the $W$ scale is too low, and not characteristic of the process. In the tail of the distribution, we expect configuration (b) to dominate, because it results in a larger second-jet $E_{T}$ for fixed center-of-mass partonic energy; contributions from higher center-of-mass energies will be suppressed by the fall-off of the parton distributions.

Can we choose a scale that treats the different final-state objects more democratically? The 
total partonic transverse energy,

$$
\hat{H}_{T}=\sum_{\text {partons } i} E_{T}^{i}+E_{T}^{e}+\boldsymbol{E}_{T}
$$

or a fixed fraction of it, is such a choice. As we can see in the right panels of figs. 1 and 2, this choice results in stable and sensible NLO predictions - and also in a relatively flat ratio of the NLO and LO predictions. For LO predictions, it is better to use such a scale when NLO results are unavailable. A similar type of scale choice, based on the combined invariant mass of the jets, has been motivated by soft-collinear effective theory [22]. Local scales associated with "branching histories" as used in parton showers have recently been studied for $W+3$-jet production at LO [19].

\section{3. $Z+$ Jets at the Tevatron}

At hadron colliders, $Z$ boson production manifests itself primarily in either charged-lepton pair production, or the production of missing transverse energy (when the $Z$ decays to neutrinos). The latter process is an important background to a wide variety of supersymmetry searches (when no charged lepton is required), and to dark matter searches more generally. The $l^{+} l^{-}$mode has a significantly lower rate, but it is an excellent calibration process, as the $Z$ can be reconstructed precisely. It is also an excellent process for confronting NLO predictions with experimental data.

We have computed the NLO $Z+1,2,3$-jet production cross sections for the Tevatron ( $p \bar{p}$ collisions at $\sqrt{s}=1.96 \mathrm{TeV}$ ), with the $Z$ decaying into a charged lepton pair. We applied the same cuts used by the CDF collaboration [20] in their measurement of these processes for $Z \rightarrow e^{+} e^{-}$,

$$
\begin{aligned}
& P_{T}^{\text {jet }}>30 \mathrm{GeV}, \quad E_{T}^{e}>25 \mathrm{GeV}, \quad \Delta R_{e-\text { jet }}>0.7, \quad 66<M_{e^{+} e^{-}}<116 \mathrm{GeV}, \\
& \left|\eta^{\text {jet }}\right|<2.1, \quad\left|\eta^{e_{1}}\right|<1, \quad\left|\eta^{e_{2}}\right|<1 \quad \text { or } \quad 1.2<\left|\eta^{e_{2}}\right|<2.8,
\end{aligned}
$$

where the electron cuts apply to both electrons and positrons, and the jet cuts apply to all jets. We cut on the jet pseudo-rapidity $\eta$ rather than CDF's cut on rapidity $y$; the two cuts coincide at LO but differ slightly at NLO. We employed three different infrared-safe jet algorithms [23], SISCone (with merging parameter $f=0.75$ ), $k_{T}$ and anti- $k_{T}$, all with $R=0.7$. Production of an $l^{+} l^{-}$pair can also be mediated by a virtual photon; we include these contributions as well, although they are suppressed by the cut on the lepton-pair invariant mass $M_{e^{+}} e^{-}$.

Fig. 4 shows how the $Z+1,2,3$-jet cross section depends on a fixed scale $\mu$, independent of the event kinematics, for the anti- $k_{T}$ algorithm and with the cuts (3.1). Here choosing $\mu \approx M_{Z}$ is appropriate, because the cross section is dominated by low- $P_{T}$ jets. The upper three panels show the scale dependence of the cross section at NLO, compared to that at LO, in $Z+1-, Z+2-$, and $Z+3-$ jet production, respectively. They illustrate the lessened dependence at NLO. The bottom panel shows the ratio of NLO to LO results for all three cases, demonstrating the increasing sensitivity to scale variations at LO with increasing number of jets. This is expected, because there is an additional power of $\alpha_{s}(\mu)$ multiplying the LO cross section for each additional jet. Accordingly, the impact of an NLO calculation also grows with the number of jets. The results for the $k_{T}$ and SISCone algorithms (not shown) are similar.

Fig. 5 compares the theoretical predictions for the second-jet $P_{T}$ distribution in $Z+2$-jet production with data from CDF [20]. CDF used the midpoint algorithm [24]. This algorithm is 


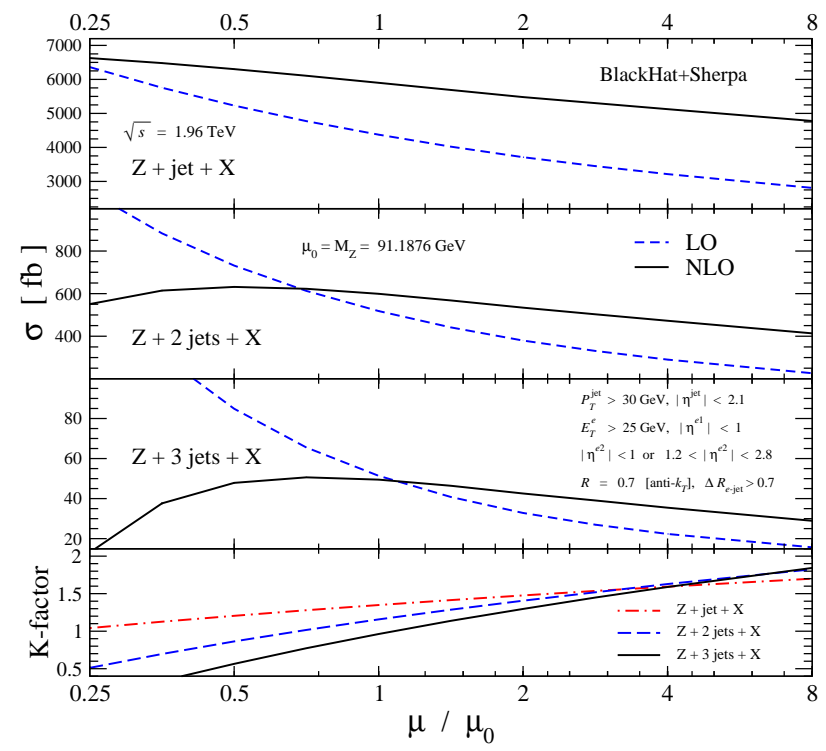

Figure 4: The scale dependence of the cross section for $Z+1,2,3$-jet production at the Tevatron, for the anti- $k_{T}$ jet algorithm using a leading-color approximation with $n_{f}$ terms, as a function of the common renormalization and factorization scale $\mu$, with $\mu_{0}=M_{Z}$. The bottom panel shows the $K$ factors, or ratios between NLO and LO results, for the three cases.

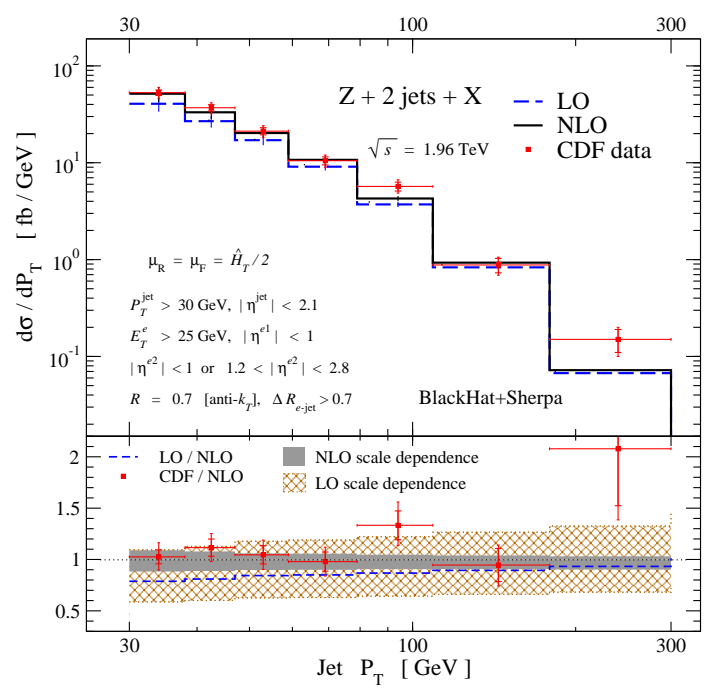

Figure 5: The second-jet $P_{T}$ distribution for $Z+2$ jets at LO and NLO compared against CDF data [20].

infrared unsafe for $Z+3$-jets at NLO, so we use infrared-safe ones instead. Fig. 5 shows results for the anti- $k_{T}$ algorithm; the other two algorithms yield similar results. It is worth noting that CDF did not attempt to "deconvolve" the hadronization corrections (estimated using Pythia) from their measured data; rather, they provided a table of hadronization corrections. This is helpful because it will allow for future improvements to hadronization models to be taken into account in theoretical predictions. Accordingly, we have used these hadronization corrections to generate a complete prediction from the LO and NLO perturbative predictions. The hadronization corrections 
are significant for low $P_{T}$, on the order of $20 \%$ at $30 \mathrm{GeV}$, and become rather small at larger jet transverse momenta. As expected, the LO scale-dependence band is much larger than the NLO one. Excepting perhaps the last bin, the agreement between the NLO prediction and the data is quite good, especially given the different jet algorithms.

Fig. 6 gives our predictions for the three jet $P_{T}$ distributions in $Z+3$-jet production, using the anti- $k_{T}$ jet algorithm. With the choice of scale $\mu=\hat{H}_{T} / 2$, only minor shape changes are visible between LO and NLO, for all three distributions. The NLO plots are based on a leading-color approximation along the lines of refs. [3, 4], except that pieces proportional to the number of light quark flavors $\left(n_{f}\right)$ are included. We expect this approximation to be valid to a few percent.

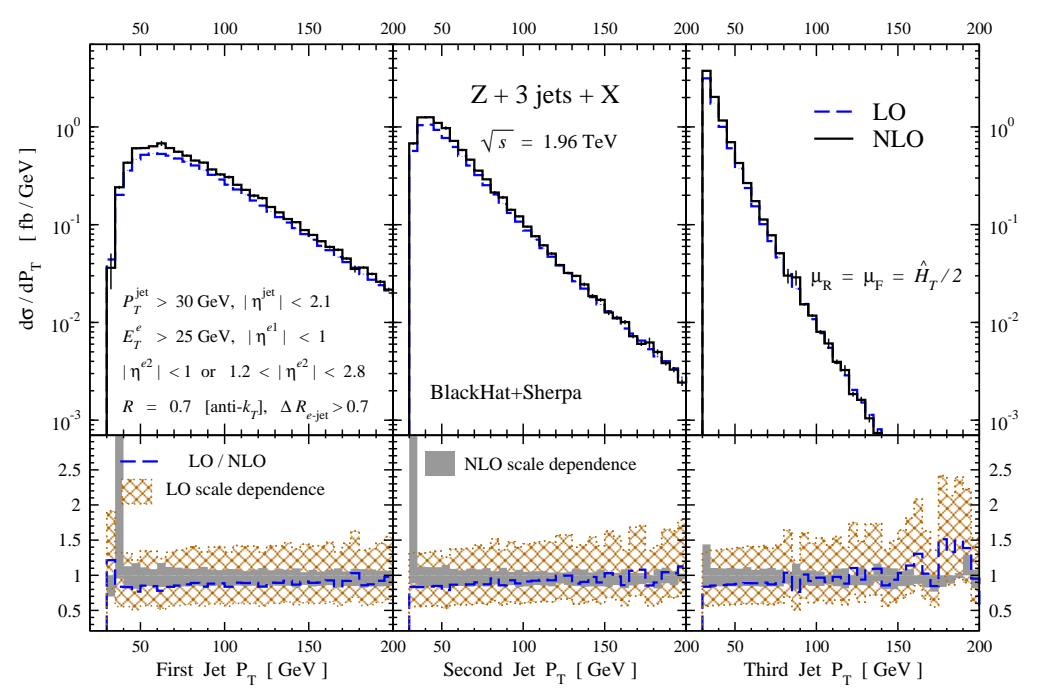

Figure 6: The LO and NLO $P_{T}$ distributions for $Z+3$-jet production for the leading, second and third jet, for the anti- $k_{T}$ algorithm and scale choice $\mu=\hat{H}_{T} / 2$. The thin vertical bars in the top panels indicate the integration errors.

\section{4. $W$ Polarization at the LHC}
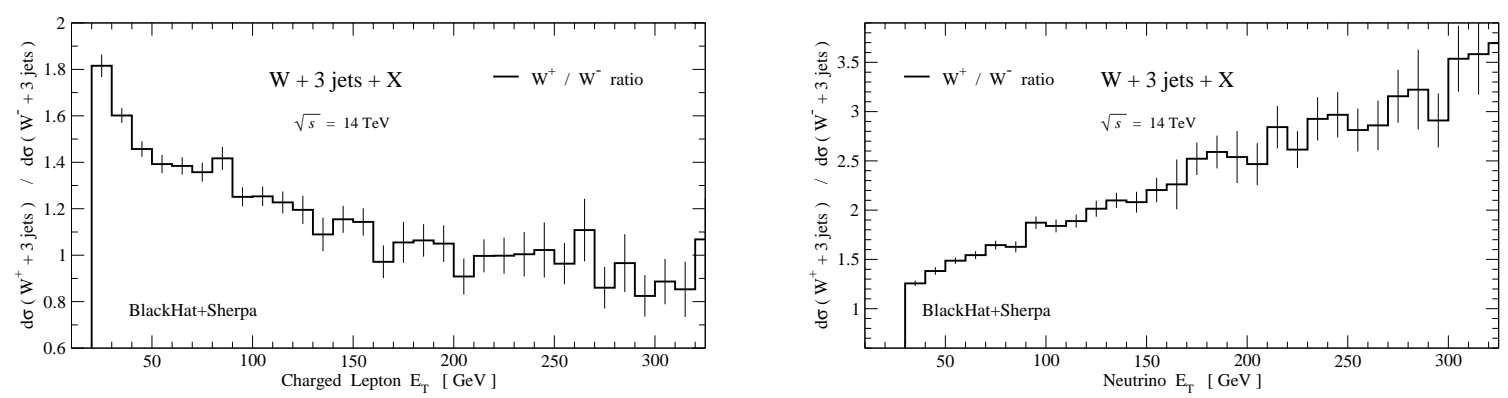

Figure 7: The left panel shows the ratio of the charged-lepton $E_{T}$ distributions at the $\mathrm{LHC}$ for $W^{+}$and $W^{-}$ production in association with at least three jets, computed at NLO. The right panel shows the corresponding ratio for the neutrino $E_{T}$, or equivalently $\mathbb{E}_{T}$.

As noted in ref. [4], at the LHC the $E_{T}$ distributions of the daughter leptons show a surprisingly strong shape dependence on whether they come from a $W^{+}$or a $W^{-}$, independent of the number 
of jets. Fig. 7 shows the ratio of the NLO transverse energy distributions for the $W^{ \pm}$boson decay products in inclusive $W+3$-jet production at the LHC, charged leptons in the left panel and neutrinos in the right panel. The differences between $W^{+}$and $W^{-}$distributions are quite dramatic. The left panel shows a large ratio for $W^{+}$to $W^{-}$at small $E_{T}^{e}$ which declines at larger $E_{T}^{e}$. In contrast, the corresponding ratio for the $E_{T}^{v}$, or equivalently the missing transverse energy $\mathbb{E}_{T}$ in the event, starts somewhat smaller but increases rapidly with $E_{T}$. The significant difference in behavior between $W^{+}$and $W^{-}$suggests a means for separating $W$ bosons produced in top quark decays from those produced from light quarks; the $W$ s from top decays do not exhibit a similar phenomenon.
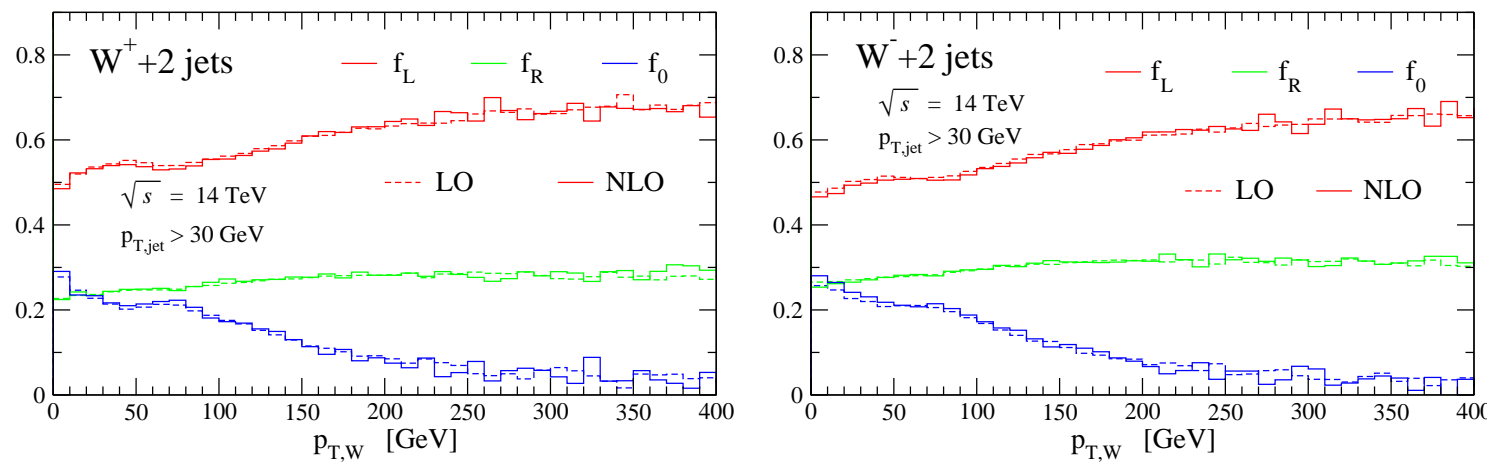

Figure 8: The LO and NLO predictions for polarization fractions of the left-handed, $f_{L}$ (top curve), righthanded $f_{R}$ (middle curve) and longitudinal $f_{0}$ (bottom curve) fractions for $W+2$ jets at the LHC. The left panel gives the polarization for $W^{+}$and the right panel for $W^{-}$. For high transverse momentum, $P_{T, W}$, the $W$ bosons become predominantly left-handed.

This disparate behavior is explained by a net left-handed polarization for both $W^{+}$and $W^{-}$at high transverse momentum. This effect is easily visible at LO, and it does not get washed out at NLO. In fig. 8, we give the fraction of $W$ bosons in each of the three polarization states, left-handed, right-handed and longitudinal $\left(f_{L}, f_{R}, f_{0}\right.$, respectively) for $W+2$-jet production at the LHC, at both LO and NLO. As seen in the figure, at high transverse momentum the $W^{ \pm}$bosons are preferentially left handed. Although the cross-sections for $W^{+}$and $W^{-}$are rather different, their polarizations are nearly identical. Interestingly, we also find that when the $W$ s have a transverse momentum of more than $50 \mathrm{GeV}$, the polarization is quite independent of the jet transverse energy cuts. With $W^{ \pm}$bosons left-hand polarized at large $E_{T}^{W}$, the $W^{+}$tends to emit the left-handed neutrino forward relative to its direction of motion (resulting in a larger transverse energy) and the right-handed positron backward (smaller transverse energy). In contrast, the $W^{-}$prefers to emit the left-handed electron forward. At high $E_{T}$, such decays produce an enhancement in the neutrino $E_{T}$ distribution and a depletion in the charged-lepton distribution, for $W^{+}$relative to $W^{-}$, consistent with the results displayed in fig. 7. We note that this phenomenon is distinct from the well-known dilution of the $W$ rapidity asymmetry at the Tevatron, when passing to the decay lepton, which can be explained using angular momentum conservation solely along the beam axis [25].

\section{Emission into Rapidity Gaps}

In previous work [21], we provided the first NLO study of the probability of emitting a third jet in $W+2$-jet events, as a function of the rapidity interval between two leading- $E_{T}$ jets at the LHC. 
This distribution was studied earlier at LO at the Tevatron and compared to CDF data [26]. Jet emission probabilities are relevant to Higgs searches in vector-boson fusion [27], in which colorsinglet exchange leads to a paucity of jet radiation in the central region between two forward tag jets. On the other hand, QCD backgrounds with color exchange, as in $W+2$-jet production, will generally lead to significant jet radiation.

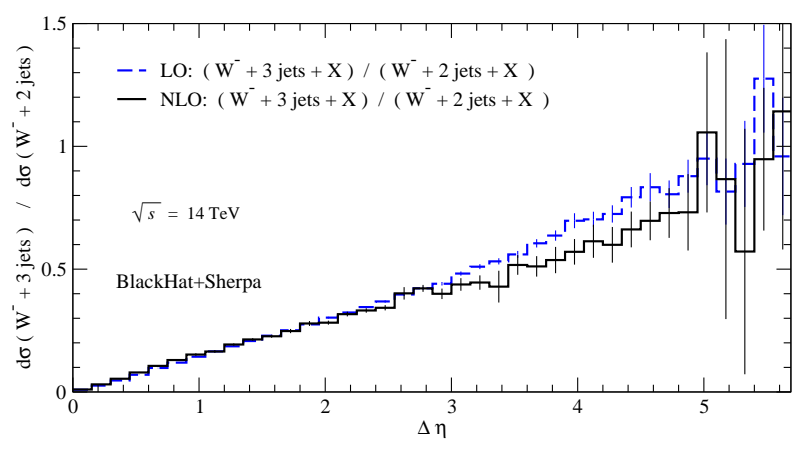

Figure 9: The ratio of the inclusive $W^{-}+3$-jet cross section to the $W^{-}+2$-jet cross section as a function of the pseudorapidity separation $\Delta \eta$ between the two most widely separated jets that pass the cuts. The solid (black) line gives the NLO result, while the dashed (blue) line gives the LO results.

To mimic vector-boson fusion searches, however, the appropriate tag jets are not the two hardest ones (by $E_{T}$ ), but rather the two most separated in pseudorapidity. Therefore, in fig. 9 we present the ratio of the $W^{-}+3$-jet cross section to the $W^{-}+2$-jet cross section as a function of the pseudorapidity separation $\Delta \eta$ between the two most separated jets. The emission probability rises roughly linearly with $\Delta \eta$. The NLO result is somewhat less than the LO one at large $\Delta \eta$. (The ratio for $W^{+}$is quite similar.) This plot is similar to one for Higgs production in association with jets [28], obtained from high-energy factorization considerations. It would be interesting to compare results obtained in this way to NLO results for the same quantities.

\section{Conclusions}

In this Contribution we presented some new results for $W+3$-jet production obtained from BLACKHAT combined with SHERPA, expanding on earlier scale-dependence studies [3, 4]. We also demonstrated that $W$ bosons produced at large $P_{T}$ are indeed polarized left-handed, explaining an asymmetry between $W^{+}$and $W^{-}$in the transverse energy distributions of the daughter leptons. Because $W$ s from top decays do not exhibit this polarization effect, it may prove effective for distinguishing such $W$ s from ones produced by light quarks. We presented the first NLO study of the probability of emitting a third jet between the two most widely separated jets in $W+2$ jet production. We also presented the first NLO results for $Z+3$-jet production. We observed that even at the Tevatron, choosing the renormalization and factorization scale to equal the vector boson transverse energy is not a particularly good choice, as it induces large shape changes between LO and NLO.

A publicly available version of BLACKHAT is in preparation and is currently being tested in diverse projects (see e.g. ref. [29]). This version uses the proposed Les Houches interface for one- 
loop matrix elements. It has been tested with both $\mathrm{C}++$ and Fortran clients. The public version will provide all processes that have been carefully tested with the full BLACKHAT code.

In the more distant future, the next benchmark process for BLACKHAT + SHERPA is the production of a $W$ boson in association with four jets at NLO. Using the techniques described above, the virtual part of the NLO cross section seems within reach. Computing the real emission matrix elements, and integrating them over the seven-particle phase space (including the decay of the vector boson) appears to be rather challenging with the current tools, due to the large number of integration channels. It is interesting to note that in this case the bottleneck no longer seems to be the virtual contributions to the cross section.

The results summarized here are indicative of the type of physics that can be carried out using BLACKHAT in conjunction with SHERPA. We look forward to comparing predictions from these tools to the forthcoming LHC data.

\section{Acknowledgments}

We thank Jeppe Andersen, Rikkert Frederix, and Markus Stoye for helpful conversations. This research was supported by the US Department of Energy under contracts DE-FG03-91ER40662, DE-AC02-76SF00515 and DE-FC02-94ER40818. DAK's research is supported by the European Research Council under Advanced Investigator Grant ERC-AdG-228301. This research used resources of Academic Technology Services at UCLA, PhenoGrid using the GridPP infrastructure, and the National Energy Research Scientific Computing Center, which is supported by the Office of Science of the U.S. Department of Energy under Contract No. DE-AC02-05CH11231.

\section{References}

[1] C. F. Berger, Z. Bern, L. J. Dixon, F. Febres Cordero, D. Forde, H. Ita, D. A. Kosower and D. Maître, Phys. Rev. D 78, 036003 (2008) [0803.4180 [hep-ph]].

[2] C. F. Berger et al., 0808.0941 [hep-ph].

[3] C. F. Berger, Z. Bern, L. J. Dixon, F. Febres Cordero, D. Forde, T. Gleisberg, H. Ita, D. A. Kosower and D. Maître, Phys. Rev. Lett. 102, 222001 (2009) [0902.2760 [hep-ph]].

[4] C. F. Berger et al., Phys. Rev. D 80, 074036 (2009) [0907.1984 [hep-ph]].

[5] T. Gleisberg, S. Höche, F. Krauss, M. Schönherr, S. Schumann, F. Siegert and J. Winter, JHEP 0902 (2009) 007 [0811.4622 [hep-ph]]; T. Gleisberg and F. Krauss, Eur. Phys. J. C 53, 501 (2008) [0709.2881 [hep-ph]].

[6] S. Catani and M. H. Seymour, Nucl. Phys. B 485, 291 (1997) [Erratum-ibid. B 510, 503 (1998)] [hep-ph/9605323].

[7] R. Kleiss and R. Pittau, Comput. Phys. Commun. 83, 141 (1994) [hep-ph/9405257].

[8] Z. Bern, L. J. Dixon and D. A. Kosower, Nucl. Phys. B 513, 3 (1998) [hep-ph/9708239]; JHEP 0408, 012 (2004) [hep-ph/0404293].

[9] R. Britto, F. Cachazo and B. Feng, Nucl. Phys. B 725, 275 (2005) [hep-th/0412103].

[10] D. Forde, Phys. Rev. D 75, 125019 (2007) [0704.1835 [hep-ph]].

[11] G. Ossola, C. G. Papadopoulos and R. Pittau, Nucl. Phys. B 763, 147 (2007) [hep-ph/0609007]. 
[12] Z. Bern, L. J. Dixon and D. A. Kosower, Phys. Rev. D 71, 105013 (2005) [hep-th/0501240]; Phys. Rev. D 72, 125003 (2005) [hep-ph/0505055]; Phys. Rev. D 73, 065013 (2006) [hep-ph/0507005]; D. Forde and D. A. Kosower, Phys. Rev. D 73, 065007 (2006) [hep-th/0507292]; Phys. Rev. D 73, 061701 (2006) [hep-ph/0509358]; C. F. Berger, Z. Bern, L. J. Dixon, D. Forde and D. A. Kosower, Phys. Rev. D 75, 016006 (2007) [hep-ph/0607014]; Phys. Rev. D 74, 036009 (2006) [hep-ph/0604195].

[13] S. D. Badger, JHEP 0901, 049 (2009) [0806.4600 [hep-ph]].

[14] Z. Bern and A. G. Morgan, Nucl. Phys. B 467, 479 (1996) [hep-ph/9511336]; Z. Bern, L. J. Dixon, D. C. Dunbar and D. A. Kosower, Phys. Lett. B 394, 105 (1997) [hep-th/9611127]; C. Anastasiou, R. Britto, B. Feng, Z. Kunszt and P. Mastrolia, Phys. Lett. B 645, 213 (2007) [hep-ph/0609191]; R. Britto and B. Feng, JHEP 0802, 095 (2008) [0711.4284 [hep-ph]]; G. Ossola, C. G. Papadopoulos and R. Pittau, JHEP 0805, 004 (2008) [0802.1876 [hep-ph]]; R. K. Ellis, W. T. Giele, Z. Kunszt and K. Melnikov, Nucl. Phys. B 822, 270 (2009) [0806.3467 [hep-ph]].

[15] W. T. Giele, Z. Kunszt and K. Melnikov, JHEP 0804, 049 (2008) [0801.2237 [hep-ph]].

[16] Y. Hida, X. S. Li and D. H. Bailey, http://crd.lbl.gov/ /dhbailey/mpdist, report LBNL-46996.

[17] A. Bredenstein, A. Denner, S. Dittmaier and S. Pozzorini, JHEP 0808, 108 (2008) [0807.1248 [hep-ph]]; Phys. Rev. Lett. 103, 012002 (2009) [0905.0110 [hep-ph]]; G. Bevilacqua, M. Czakon, C. G. Papadopoulos, R. Pittau and M. Worek, JHEP 0909, 109 (2009) [0907.4723 [hep-ph]]; T. Binoth, N. Greiner, A. Guffanti, J. P. Guillet, T. Reiter and J. Reuter, 0910.4379 [hep-ph].

[18] R. K. Ellis, K. Melnikov and G. Zanderighi, Phys. Rev. D 80, 094002 (2009) [0906.1445 [hep-ph]].

[19] K. Melnikov and G. Zanderighi, 0910.3671 [hep-ph].

[20] T. Aaltonen et al. [CDF Collaboration], Phys. Rev. Lett. 100, 102001 (2008) [0711.3717 [hep-ex]].

[21] T. Aaltonen et al. [CDF Collaboration], Phys. Rev. D 77, 011108 (2008) [0711.4044 [hep-ex]].

[22] C. W. Bauer and B. O. Lange, 0905.4739 [hep-ph].

[23] S. Catani, Y. L. Dokshitzer, M. H. Seymour and B. R. Webber, Nucl. Phys. B 406, 187 (1993); S. D. Ellis and D. E. Soper, Phys. Rev. D 48, 3160 (1993) [hep-ph/9305266]; G. P. Salam and G. Soyez, JHEP 0705, 086 (2007) [0704.0292 [hep-ph]]; M. Cacciari, G. P. Salam and G. Soyez, JHEP 0804, 063 (2008) [0802.1189 [hep-ph]]; G. P. Salam, 0906.1833 [hep-ph].

[24] R. Akers et al. [OPAL Collaboration], Z. Phys. C 63, 197 (1994); M. H. Seymour, Nucl. Phys. B 513, 269 (1998) [hep-ph/9707338]; A. Abulencia et al. [CDF Collaboration], Phys. Rev. D 74, 071103 (2006) [hep-ex/0512020].

[25] R. K. Ellis, W. J. Stirling and B. R. Webber, $Q C D$ and Collider Physics (Cambridge University Press, 1996).

[26] J. M. Campbell, J. W. Huston and W. J. Stirling, Rept. Prog. Phys. 70, 89 (2007) [hep-ph/0611148].

[27] D. Zeppenfeld, R. Kinnunen, A. Nikitenko and E. Richter-Was, Phys. Rev. D 62, 013009 (2000) [hep-ph/0002036]; M. Dührssen, S. Heinemeyer, H. Logan, D. Rainwater, G. Weiglein and D. Zeppenfeld, Phys. Rev. D 70, 113009 (2004) [hep-ph/0406323].

[28] J. R. Andersen, V. Del Duca and C. D. White, JHEP 0902, 015 (2009) [0808.3696 [hep-ph]]; J. R. Andersen, these proceedings.

[29] R. Frederix, in these proceedings. 